\title{
CAPÍTULO 07: ANÁLISE DE DEFEITOS NO ROLAMENTO EM UM MOTOR DE INDUÇÃO TRIFÁSICO UTILIZANDO A EMISSÃO ACÚSTICA
}

\section{CAPÍTULO 07: ANÁLISIS DE LOS DEFECTOS DE LOS RODAMIENTOS EN UN MOTOR DE INDUCCIÓN TRIFÁSICO CON EMISIÓN ACÚSTICA}

\author{
CHAPTER 07: ANALYSIS OF BEARING DEFECTS IN A THREE-PHASE \\ INDUCTION MOTOR USING ACOUSTIC EMISSION
}

\author{
Gustavo Pereira Bruno; André Fellipe Cavalcante Silva²; José Anselmo de Lucena Júnior ${ }^{3}$; Silvana Luciene do \\ Nascimento Cunha Costa ${ }^{4}$
}

DOI: https://doi.org/10.31692/978-65-88970-06-5.99-115

\begin{abstract}
RESUMO
Os motores de indução trifásicos (MIT) são responsáveis por $90 \%$ da fonte primária de energia mecânica que é utilizada nas indústrias. Para garantir o desempenho desejado destes equipamentos, sem que haja interrupções na linha de produção, faz-se necessária a elaboração e a implementação de um plano de manutenção. Dentre as práticas de manutenção, ressalta-se a manutenção preditiva, que é de suma importância para otimizar os processos industriais e poupar custos operacionais. Para viabilizar este tipo de manutenção em motores de indução trifásico (MIT), é necessária a utilização de um sensor para aferir uma grandeza física e realizar a predição. Neste trabalho foi proposto a utilização de um sensor alternativo (microfone) para detectar falhas na pista interna e na pista externa de um rolamento. Com esta técnica alternativa é possível realizar o diagnostico sem qualquer tipo de contato físico com o motor, tornando viável a utilização de um único dispositivo para diversos motores. A execução do processamento do áudio é realizada de forma sequencial e automática. Com isto, para diagnosticar o rolamento, o usuário tem a função única de iniciar o sistema e aguardar o resultado. Para identificar o tipo do defeito, dentre os estudados, foi utilizada uma Rede Neural Convolucional (RNC), que ao final do treinamento, obteve uma acurácia de 93,02\%.
\end{abstract}

Palavras-Chave: Emissão Acústica; MIT; RNC.

\section{RESUMEN}

Los motores de inducción trifásica (MIT) representan el $90 \%$ de la fuente primaria de energía mecánica que es utilizada en las industrias. Para garantizar el desempeño deseado de estos equipos, sin que haya interrupciones en la línea de producción, se hace necesaria la elaboración e implementación de un plan de mantenimiento. Dentro de las prácticas de mantenimiento, se destaca el mantenimiento predictivo, que es de suma importancia para optimizar los procesos industriales y disminuir los costos operacionales. Para hacer posible este tipo de mantenimiento en motores de inducción trifásica (MIT), es necesaria la utilización de un sensor para medir una cantidad física y realizar una predicción. En este trabajo se propuso la utilización de un sensor alternativo (micrófono) para detectar fallas en la pista interna y en la pista externa de un rodamiento. Con esta técnica alternativa es posible realizar un diagnóstico sin ningún tipo de contacto físico con el motor, haciendo posible la utilización de un único dispositivo para diversos motores. El procesamiento del audio se realiza de forma secuencial y automática. Con esto, para diagnosticar un rodamiento, el usuario tiene la única función de iniciar el sistema y aguardar el resultado. Para identificar el tipo de defecto, entre los estudiados, se utilizó una Red Neural Convolucional (RNC), que al final del entrenamiento, obtuvo una precisión del 93,02

Palabras clave: emisión acústica; MIT; RNC.

ABSTRACT

Three-phase induction motors (TIM) are responsible for $90 \%$ of the primary source of mechanical

${ }^{1}$ Mestrado em Engenharia Elétrica, IFPB, gustavo.bruno@academico.ifpb.edu.br

${ }^{2}$ Doutor, IFPB, andrefellipecs@gmail.com

${ }^{3}$ Mestrado em Engenharia Mecânica, UFPB

${ }^{4}$ Doutor, IFPB, silvana@ifpb.edu.br 
energy that is used in industries. To guarantee the desired performance of this equipment, without interruptions in the production line, it is necessary to develop and implement a maintenance plan. Among maintenance practices, predictive maintenance stands out, which is of paramount importance to optimize industrial processes and save operating costs. To make this type of maintenance feasible in three-phase induction motors (TIM), it is necessary to use a sensor to measure a physical quantity and make the prediction. In this work it was proposed to use an alternative sensor (microphone) to detect failures in the inner and outer race of a bearing. With this alternative technique it is possible to carry out the diagnosis without any kind of physical contact with the engine, making it feasible to use a single device for several engines. The audio processing is performed sequentially and automatically. With this, to diagnose the bearing, the user has the unique function of starting the system and waiting for the result. To identify the type of defect, among those studied, a Convolutional Neural Network (CNN) was used, which at the end of the training, obtained an accuracy of 93.02\%.

Keywords: TIM; Acoustic Emission; CNN.

\section{INTRODUÇÃO}

Motores de indução trifásicos (MIT) consomem cerca de $40 \%$ a $50 \%$ de toda energia elétrica no setor industrial, sendo responsáveis por mais de $90 \%$ de todo acionamento das principais nações industrializadas. Apesar da natureza simplista e robusta desta máquina, é necessário que haja um plano de manutenção para garantir sua integridade física e boa performance (AHAMED et al., 2010). Dentre os tipos de manutenção existentes, conforme a NBR 5462, se destaca a manutenção preditiva, onde o elemento em questão é monitorado constantemente, permitindo assim, um uso otimizado de sua vida útil e baixa probabilidade de uma parada não planejada.

Conforme Silva (2018), existem sinais acústicos quando há alguma variação na pressão do ar, em relação à pressão média do ambiente, ao longo de um determinado tempo. O diagnóstico por emissão acústica (EA) está ganhando espaço no cenário atual por meio de inovações tecnológicas e pesquisas dirigidas. Esta técnica alternativa permite um diagnóstico para detecção de falhas em motores na fase inicial (PODDAR e TANDON, 2019), além de admitir uma ampla faixa de velocidade, uma vez que o método convencional (análise por vibrações) exige que o motor esteja funcionando na velocidade nominal, para que o sinal de vibração tenha energia suficiente para ser analisada de forma satisfatória (AKBEN, 2019).

A técnica de análise por emissão acústica para detectar defeitos em rolamentos é amplamente estudada por diferentes pesquisadores, que em geral, realizam a análise no domínio da frequência.

Este trabalho se propõe a utilizar o processamento digital de imagens (PDI) para tratar o espectrograma do sinal de áudio emitido pelo motor em funcionamento. A partir da análise do espectrograma será feito um diagnóstico do estado do motor, indicando se há falha nos rolamentos de pista interna e externa. 


\section{FUNDAMENTAÇÃO TEÓRICA}

O MIT é um elemento conversor de energia elétrica em energia motriz mais utilizado, sendo indispensável aos processos produtivos industriais, porque, além do baixo custo, apresentam adaptabilidade às diferentes exigências de cargas mecânicas e resistência para operação em ambientes (SANTOS et al., 2014). Ainda que o MIT disponha de extrema robustez e confiabilidade, com o decorrer do tempo ocorrerá naturalmente o desgaste de seus elementos construtivos. Dentre estes elementos, o que se destaca são os rolamentos devido à ação do atrito. Segundo estudos, cerca de 50\% dos defeitos relatados em MITs ocorrem no rolamento (SALAZAR-VILLANUEVA e IBARRA-MANZANO, 2013).

Defeitos incipientes (falhas potenciais) podem ser detectados, antes de se tornaram falhas significativas (falhas funcionais). Quando um defeito não é detectado, o processo natural de evolução da falha levará à instalação de uma falha potencial, a qual acarretará, necessariamente, em prejuízos ao processo industrial.

Um rolamento perde sua funcionalidade quando instalado ou mantido inadequadamente. Nesta situação, o rolamento apresentará uma falha prematura, totalmente diferente de uma falha por fadiga do aço (escamamento). Entretanto, todo tipo de falha que ocorra no rolamento terá um ruído acústico característico (NSK, 2008).

De forma elementar, um rolamento é constituído por três partes: elemento rolante, pista interna e pista externa. Considerando uma velocidade fixa e conhecida, é possível calcular a frequência com que o elemento rolante passa sobre um determinado ponto. Desta forma, um defeito genérico (um furo, por exemplo) gera componentes em uma frequência determinável. Estas frequências podem ser calculadas pelas Equações 1 e 2 [7]. A Equação 1 representa a Frequência de falha na pista interna e a Equação 2 a frequência de falha na pista externa).

$$
\begin{aligned}
& F p i=\frac{n F_{r}}{2}\left[1-\frac{d}{D} \cos (\phi)\right], \\
& F p o=\frac{n F_{r}}{2}\left[1+\frac{d}{D} \cos (\phi)\right],
\end{aligned}
$$

onde $d$ é o diâmetro do elemento rolante, $\mathrm{D}$ a distância entre centros das esferas (Pitch), $n$ o número de elementos rolantes e $\phi$ o contato angular da esfera.

Estas variáveis são parâmetros inerentes à construção do rolamento e podem ser observados na Figura 1. 
Figura 01: Geometria de um rolamento.

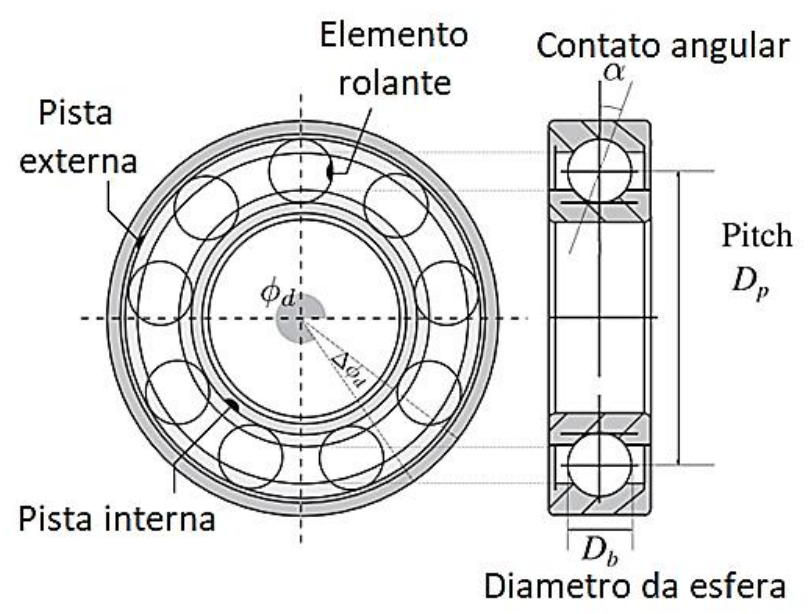

Fonte: Dolenc et al. (2015) [adaptado].

\section{METODOLOGIA}

Esta pesquisa é de caráter experimental quantitativo. O fluxograma da Figura 2 apresenta o método empregado nesta pesquisa para realizar a aquisição e o processamento do sinal.

Inicialmente, o sinal acústico do MIT é capturado pelo microfone, que é processado pela interface de áudio. Neste ponto o áudio é digitalizado e enviado para a Raspberry Pi, que direciona o áudio para um servidor online, onde é mantido um banco de dados com os áudios originais e para uma workstation, onde de fato é realizado o todo o processamento. $\mathrm{O}$ resultado deste processamento é retornado para a Raspberry $\mathrm{Pi}$, para ser exibido ao operador em um display e encaminhado também para o servidor, para ser armazenado.

Figura 02: Fluxograma do sistema proposto.

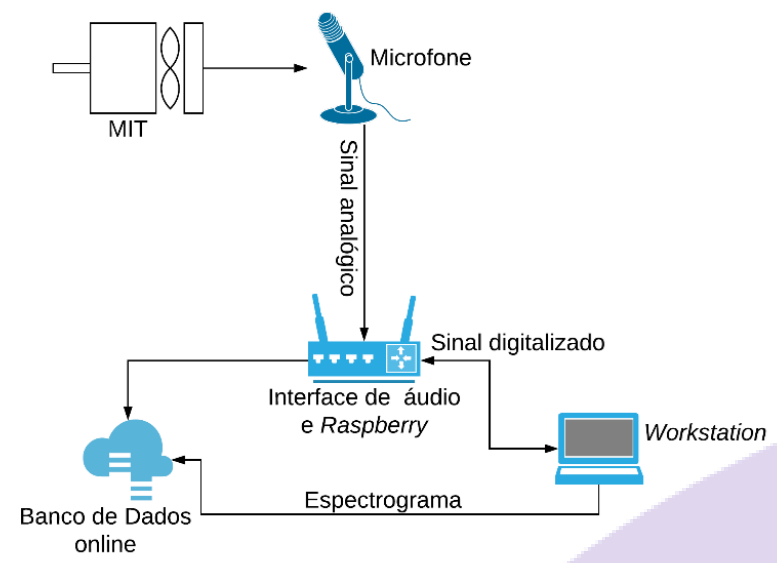

Fonte: Própria (2020). 
O microfone utilizado para aquisição do sinal gerado pelo MIT está ligado à placa de áudio (conversor $\mathrm{AD}+$ amplificador) por cabo (padrão XLR blindado). A placa de áudio está isolada eletricamente de outros dispositivos (exceto o microfone) e da rede elétrica, evitando assim ruído externo de origem eletromagnética. Além disto, esta configuração facilita o manuseio do equipamento, uma vez que o mesmo pode ser acionado remotamente, através de uma conexão sem fio (Wi-Fi).

Foi desenvolvido um sistema offline, para análise dos sinais coletados, o qual permite o armazenamento dos dados originais coletados, tanto em dispositivos físicos como cartões de memória, quanto em meios virtuais, como os serviços de armazenamento em nuvens.

Para a realização da pesquisa, foi utilizada a bancada de testes, projetada e construída pelo Grupo de Instrumentação, Controle e Estudo em Energia e Meio Ambiente (GPICEEMA), da Universidade Federal da Paraíba (UFPB), Campus João Pessoa. Nesta bancada é possível substituir o rolamento do motor e submeter os mesmos a diferentes situações de funcionamento do MIT, como por exemplo, a alteração de cargas e de velocidade sobre o eixo do motor. A bancada possui um sistema de monitoramento embarcado, que inclui um tacômetro e uma célula de carga (torquímetro). A referida bancada será descrita, detalhadamente, no item 2 .

\section{Componentes para aquisição do sinal de Emissão Acústica}

Neste tópico serão detalhados os dispositivos necessários para realização desta pesquisa.

\subsection{Microfone}

Em uma pesquisa deste tipo, que se propõe a utilizar o método da Emissão Acústica (EA), para detecção de defeitos, o dispositivo responsável pela aquisição das ondas sonoras assume um papel fundamental. O microfone exercerá a função do transdutor responsável por transformar o sinal mecânico, modulado nas ondas sonoras, em sinal elétrico a fim de que, posteriormente, seja digitalizado e analisado. A escolha de um dispositivo adequado tem influência direta sobre os procedimentos de tratamento de dados, podendo impactar e até mesmo delimitar a eficácia do sistema.

Conforme Silva (2018), existem diferentes tipos de microfones. Os que captam sons provenientes de todas as direções são chamados de omnidirecionais. Os que captam sons provenientes de uma única direção são chamados de direcionais. Os que captam sons vindos de direções opostas são chamados de bidirecionais. Existem ainda os que captam, com precisão, os sons que vêm da frente para onde aponta o transdutor. Estes microfones 
especializados, que captam apenas os sons frontais, são chamados de cardioides ou hipercardioides.

Visando a adequada captura da EA, proveniente dos componentes do rolamento do MIT, buscou-se utilizar um transdutor, cujo espectro de sensibilidade abrangesse as faixas de frequência de EA, dos itens analisados. Com base nestes parâmetros, verificou-se que o melhor transdutor indicado, para esta situação, seria um microfone do tipo condensador, que apresentasse um diafragma largo, proporcionando boa sensibilidade, com a característica de captar, com precisão, os sons provenientes da fonte emissora, para o qual o transdutor fosse apontado. Optou-se, então, por se utilizar o microfone condensador Samson Co1, com diafragma de $19 \mathrm{~mm}$, sensibilidade de $-33 \mathrm{~dB} / \mathrm{Pa}$, o qual é capaz de atuar em ampla faixa de frequências $(20 \mathrm{~Hz}$ a $18 \mathrm{kHz})$ e de operar em uma zona de captação unidirecional (polar cardioide). Estas configurações, imprescindíveis para o desenvolvimento desta pesquisa, são apresentadas na Figura 3, assim como o microfone utilizado.

Figura 03: Dispositivo de captura do sinal acústico.

A- Resposta em frequencia

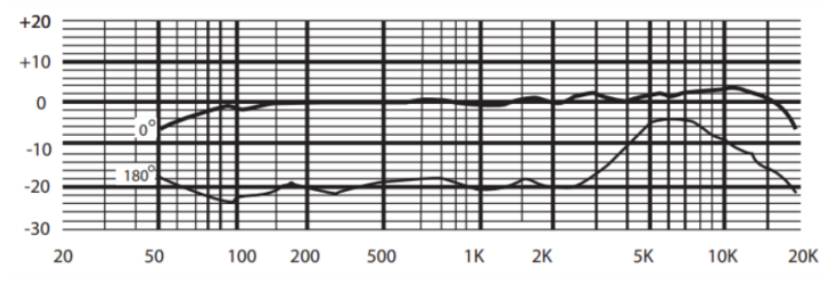

C- Microfone Samson C01
B- ângulo de operação

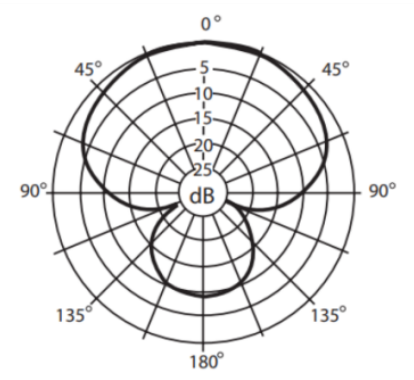

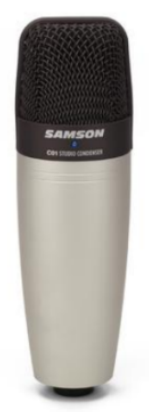

Fonte: Própria (2020).

\subsection{Interface de Áudio}

A onda sonora, captada pelo transdutor (microfone), foi transformada, por este, em um sinal elétrico analógico. A Interface de Áudio foi utilizada para realizar a conversão do sinal analógico proveniente da saída do microfone, para sinal digital. Ou seja, exerceu a 
funcionalidade de um conversor analógico digital (A/D), visando viabilizar o posterior processamento, classificação e armazenamento do sinal.

Na presente pesquisa, utilizou-se uma interface Behringer U-PHORIA UMC22, a qual possui uma taxa de amostragem máxima de $48 \mathrm{kHz}$, com resolução de 16 bits. Este dispositivo, por meio de portas $I / O$ do tipo $U S B$ permitiu a gravação do áudio diretamente em um banco de dados na Raspberry de comunicação, de onde é automaticamente enviado, via Wi-Fi, para o computador (workstation).

Neste trabalho, o áudio foi gravado a uma taxa de amostragem de 44.100 amostras por segundo, o que permitiu a digitalização de sinais de até $22 \mathrm{kHz}$, com fidelidade. Este dispositivo está ilustrado na Figura 4.

Figura 04: Interface de áudio.

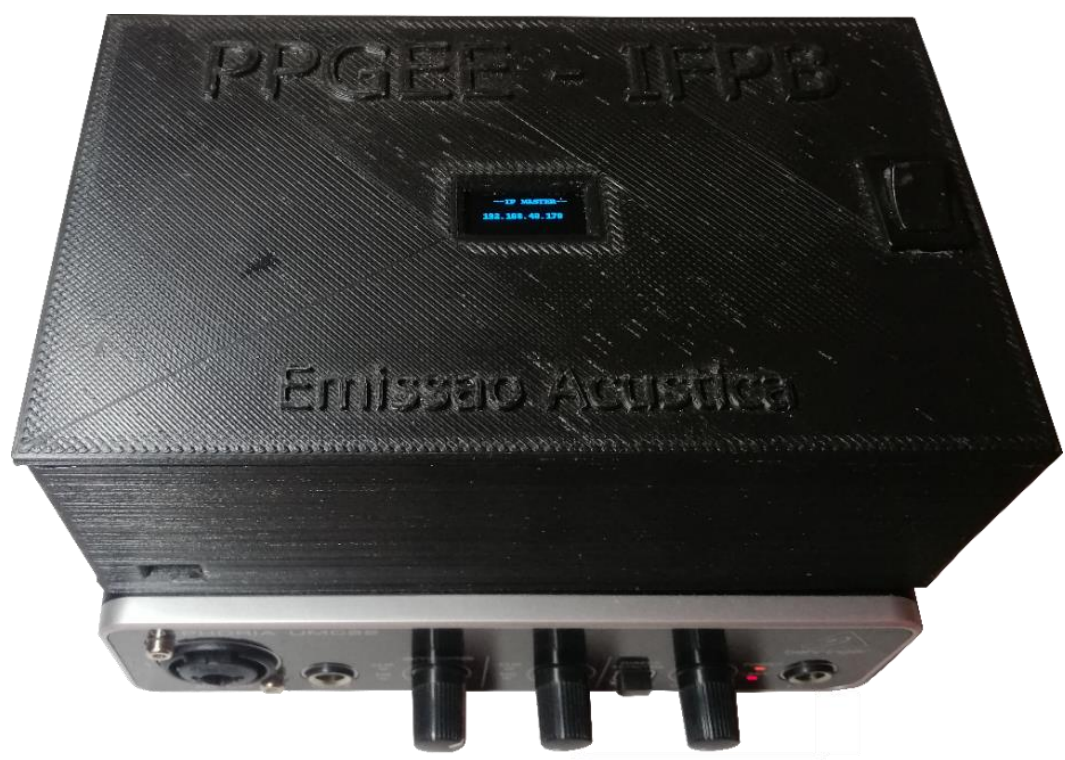

Fonte: Própria (2020).

\section{Bancada de ensaios}

A bancada utilizada nesta pesquisa foi projetada e montada pelo Grupo de Instrumentação e Controle em Estudo e Energia e Meio Ambiente (GPICEEMA) para ensaiar diferentes tipos de falhas/defeitos em motores de indução trifásicos. A bancada pode ser yista na Figura 5. É composta por: motor de indução trifásico (1), mancal para apoio con dois rolamentos (2), torquímetro digital (3), motor Corrente Contínua (CC) para aplicar carga (4) e transdutor de velocidade (5). 
Figura 05: Bancada de ensaios.

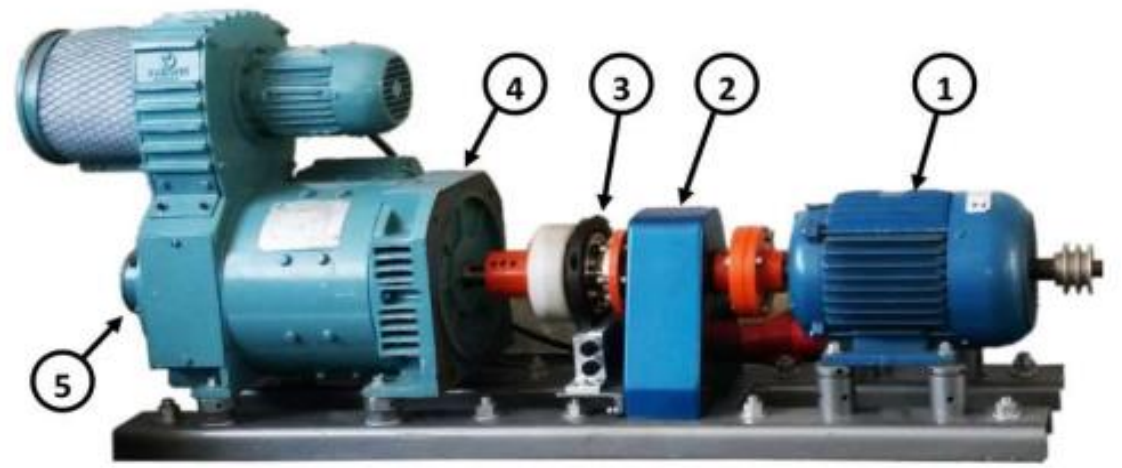

Fonte: Sobrinho (2020).

\section{Método para aquisição do sinal}

Foram utilizados quatro rolamentos do mesmo modelo/fabricante para esta pesquisa. Dentre estes quatro, três com defeitos e um saudável (controle). Os defeitos foram fabricados no laboratório da UFPB, pelo processo de eletro erosão (furo radial com $1 \mathrm{~mm}$ de diâmetro).

Para simular diferentes situações, a qual um rolamento é exposto, foi realizada a coleta dos dados em três situações diferentes para cada rolamento: com carga no eixo nula $(0 \mathrm{Nm})$, carga nominal $(12 \mathrm{Nm})$ e carga máxima $(24 \mathrm{Nm})$. Todos os ensaios foram gravados com uma duração de 60 segundos nas configurações citadas no tópico 1.2. O microfone foi posicionado a uma distância de aproximadamente $150 \mathrm{~mm}$, direcionado para a fonte emissora.

\section{Análise do defeito (pista interna e externa)}

A análise do espectrograma permite avaliar a amplitude da frequência em função do tempo. Para esta pesquisa, este tipo de análise permite acompanhar a frequência de defeito e suas harmônicas (Equações 1 e 2) em toda extensão do sinal, asseverando assim uma maior confiabilidade na análise, visto que ruídos de curta duração no tempo podem ser isolados para não interferir no diagnóstico. Além disto, por meio desta análise, é possível obter resultados confiáveis mesmo em velocidades baixas (menor que $200 \mathrm{rpm}$ ), diferente das técnicas convencionais que são ineficazes para análise em baixas velocidades (HASAN, ISLAM e KIM, 2019).

\subsection{Processamento digital da imagem}

Para realizar o diagnóstico do rolamento, o sinal acústico foi processado utilizando uma cadeia de técnicas, que são ilustradas na Figura 06 e particularizadas nos tópicos seguintes. 
Figura 06: Fluxograma do processamento digital da imagem.

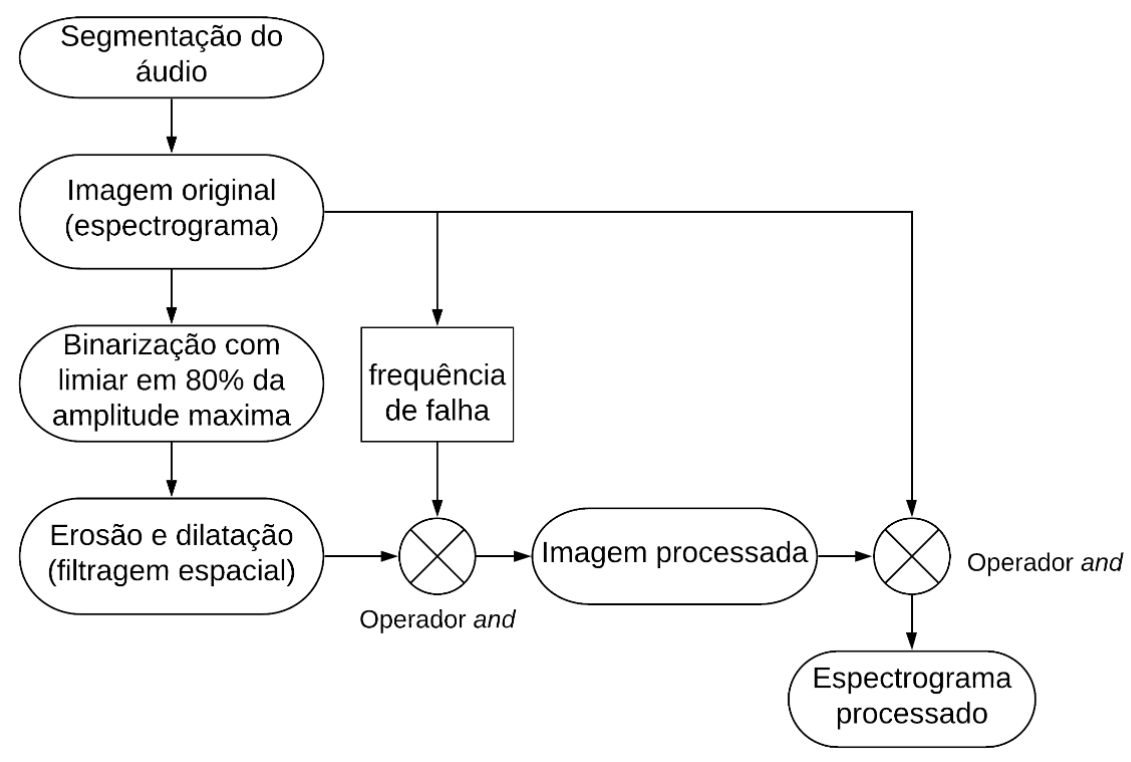

Fonte: Própria (2020).

\subsubsection{Segmentação do áudio}

Inicialmente o áudio é segmentado com overlap de 50\%, transformando assim um áudio de 60 s em cinco áudios com 20s de duração. Este procedimento foi importante para aumentar a quantidade de amostras no treinamento da rede neural convolucional. Além disto, esta técnica permite que um único áudio seja processado cinco vezes de forma independente. Com isso, caso haja algum tipo de ruído externo de curta duração no tempo, que comprometa totalmente o diagnóstico, o segmento correspondente a este ruído será descartado.

\subsubsection{Cálculo do espectrograma}

Nesta etapa é calculada a transformada rápida de Fourier (FFT) e o espectrograma do sinal. Para isto, foi utilizada a janela de Hammig com 4096 amostras. A imagem resultante é salva em formato PNG sem compactação em duas cópias: uma colorida para melhorar a experiência visual do usuário; e outra em tons de cinza para otimizar o processamento. Já espectro do sinal é utilizado para calcular as frequências de defeito, conforme as Equações 1 e 2. Estas frequências são armazenadas para serem utilizadas nas etapas posteriores

\subsubsection{Binarização da imagem}

Esta é uma técnica convencionalmente utilizada no processamento digital de imagens para realizar a segmentação de uma imagem. Nesta pesquisa, esta técnica foi utilizada para extrair da imagem original as frequências de alta magnitude. Na imagem, estas frequências 
são proporcionais ao valor do pixel onde ocorrem, ou seja, quanto mais perto do branco (255) maior a magnitude da frequência. De forma experimental, foi estipulado um limiar de 210 para realizar a Binarização, conforme a Equação 3.

$$
g(x, y)=\left\{\begin{array}{c}
0 \text { se } f(x, y) \leq 210 \\
255 \text { se } f(x, y)>210
\end{array}\right.
$$

\subsubsection{Filtragem (Erosão e Dilatação)}

O resultado da operação anterior é submetido a mais duas técnicas em conjunto, para atenuar os ruídos que possam existir e realçar as componentes de alta magnitude que ocorrem no decorrer do tempo. Pra isto, primeiro é realizado o processo de erosão com três interações, seguido do processo de dilatação com cinco interações. Foi utilizado um elemento estruturante (Kernel) em forma de linha horizontal nos dois procedimentos. Este foi um diferencial importante para esta pesquisa, pois permitiu realçar as componentes de alta magnitude que se estendem no tempo (componentes de frequências importantes para esta pesquisa) e atenuar componentes de alta magnitude com curta duração do tempo (característico de ruídos externos). Matematicamente, estas duas operações são translações de um dado conjunto numérico sobre outro e são representados conforme as Equações 4 e 5, respectivamente.

$$
\begin{aligned}
& A \ominus B=\left\{x \mid(B)_{x} \subseteq A\right\} \\
& A \ominus B=\left\{x \mid(B)_{x} \subseteq A\right\}
\end{aligned}
$$

\subsubsection{Identificação da frequência de defeito}

Esta etapa utiliza as frequências de defeito, que foram calculadas na segunda etapa, para ressaltar as frequências de alta magnitude que ocorrem na frequência de defeito e em suas harmônicas. Para isto, é criada uma máscara com linhas em branco no local correspondente às frequências que estas representam. Por sua vez, esta máscara é multiplicada com a imagem filtrada na etapa anterior. Devido a esta pesquisa estudar dois tipos de defeitos, neste procedimento são criadas duas imagens, sendo a primeira o resultado da multiplicação da imagem filtrada com a máscara contendo as frequências de defeito da pista interna e a segunda utiliza a mesma imagem filtrada multiplicada com a máscara com as frequências de defeito da pista externa. Além das frequências de defeito, ambas as máscaras contêm um retângulo preto no inferior, no local correspondente as frequências de zero a $500 \mathrm{~Hz}$. Este artifício é utilizado para realizar uma filtragem no domínio da frequência, uma vez que esta faixa de frequência não contém informações relevantes para o diagnóstico do rolamento com 
esta técnica.

Ainda nesta etapa do processamento é calculada a porcentagem de ocorrência de cada defeito. Este cálculo é o somatório dos pixels das duas imagens processadas, dividido pela somatória dos pixels da imagem filtrada. Logo tem-se dois índices: porcentagem de ocorrência na pista interna e a porcentagem de ocorrência na pista externa. Esses dois índices são utilizados para identificar se um dado rolamento possui defeito ou não. Para realizar este primeiro diagnostico, foi estabelecido experimentalmente um limar de $10 \%$. Portanto, se a somatória dos dois índices for superior a $10 \%$ indica que o rolamento está com algum tipo de defeito.

Se for detectado defeito no rolamento, as duas imagens processadas são combinadas em uma única imagem com dois canais para serem apresentadas à rede neural convolucional. Esta etapa será detalhada no tópico seguinte

\subsection{Rede Neural Convolucional}

A RNC (Rede Neural Convolucional) é uma variação das redes neurais de múltiplas camadas adaptada para ser utilizada em imagens. Este tipo de rede neural é amplamente utilizado para reconhecimento em imagens por ser capaz de aplicar diferentes filtros mantendo a disposição dos pixels, ou seja, além do valor que o pixel assume é considerado também o espaço que este se encontra, de acordo com sua vizinhança. Diferente da rede neural perceptron, na rede utilizada nesta pesquisa apenas um conjunto de entradas é conectada a cada neurônio para realizar a análise de campos receptivos locais.

Para o desenvolvimento desta pesquisa, foi utilizado o framework Keras para criar a rede neural convolucional. Esta aplicação utiliza o TensorFlow como backend engine para realizar o treinamento da rede. Com estas ferramentas, a arquitetura da rede foi elaborada de forma experimental, com duas camadas de convolução seguida de quatro camadas densas. Foi utilizada a função de ativação Relu (Equação 6) para otimizar o processamento (Krizhevsky et al. 2012). Já na camada de saída, foi utilizada a função de ativação sigmoide (funçã́o logística, Equação 7).

$$
\begin{gathered}
\operatorname{ReLU}(x)=\max \{0, x\} \quad \operatorname{ReLU}^{\prime}(x)=\left\{\begin{array}{c}
1, \text { se } x \geq 0 \\
0, \text { c.c. }
\end{array}\right. \\
\sigma(x)=\frac{1}{1+e^{x}} \quad \sigma^{\prime}(x)=\sigma(x)(1-\sigma(x))
\end{gathered}
$$

Esta arquitetura de rede foi treinada com supervisionamento utilizando um conjunto de 180 imagens, sendo estas organizadas em três classes: rolamento saudável, rolamento com 
defeito na pista interna e rolamento com defeito na pista externa.

\section{RESULTADOS E DISCUSSÃO}

Neste tópico são apresentados os resultados obtidos ao utilizar o classificador aqui desenvolvido para diagnosticar 10 áudios, sendo estes de rolamentos com defeito ou não. Ao realizar a aquisição do sinal, o motor estava funcionando na velocidade da rede $(60 \mathrm{~Hz})$, com três situações de carga mecânica imposta ao eixo: 0 Nm, 12 Nm e 24 Nm.

Já no tópico 1 serão apresentados os resultados pertinentes ao processamento digital de imagem, ao passo que no tópico 2 serão expostos os resultados da RNC.

Para simular o defeito de forma genérica no rolamento foi retirada uma pequena quantidade de material utilizando uma descarga elétrica. Com este método, foi possível fabricar o defeito de forma pontual com $1 \mathrm{~mm}$ de diâmetro, como apresentado pela Figura 7.

Figura 07: Defeito na pista interna.
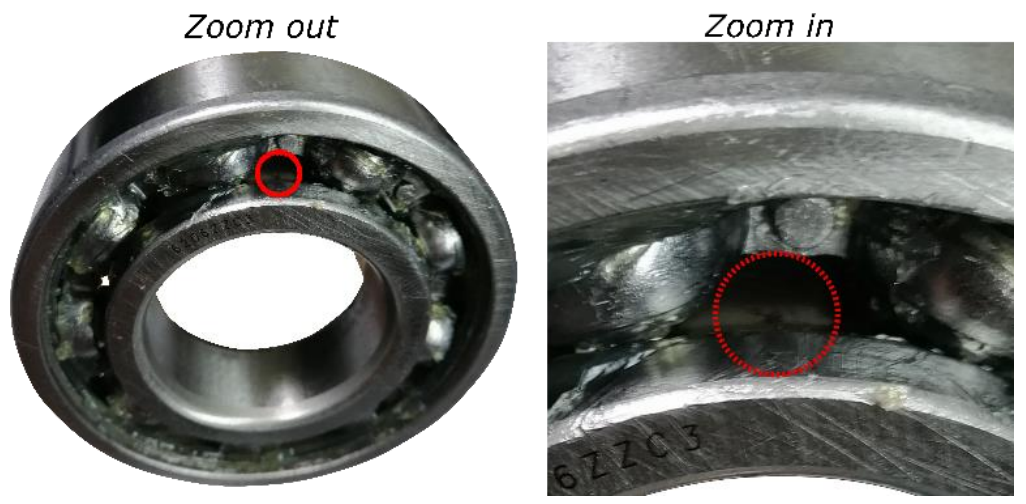

Fonte: Própria (2020).

Este método foi utilizado tanto para fabricar defeito na pista interna como na pista externa. Contudo, para cada rolamento foi fabricado só um tipo de defeito. Para assegurar que o rolamento só possuía o defeito que havia sido fabricado, foram utilizados rolamentos novos lubrificados conforme as especificações do fornecedor.

Para validar o software desenvolvido, foi utilizado um banco de dados contendo 36 áudios de rolamentos em funcionamento. A aquisição dos sinais deste banco de dados se deu conforme o tópico 1.2. Contudo, estes áudios não foram utilizados em nenhuma etapa do treinamento da rede. Os resultados desta etapa de validação serão apresentados de forma resumida pelas Figuras 8 a 10 . 
Figura 08: Diagnóstico de um rolamento saudável.

A-Audio 1

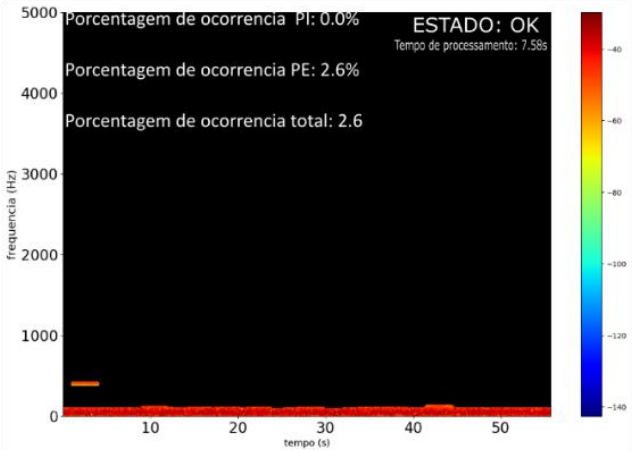

C- Audio 3

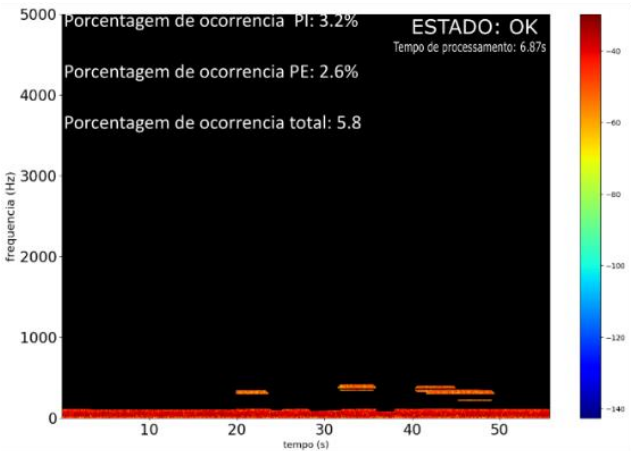

E- Audio 5

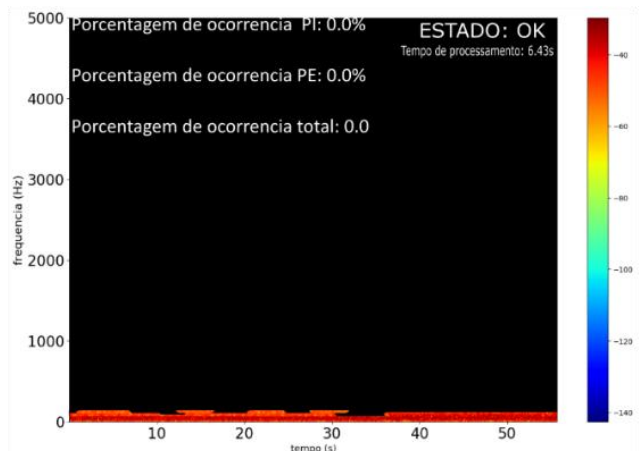

B- Audio 2

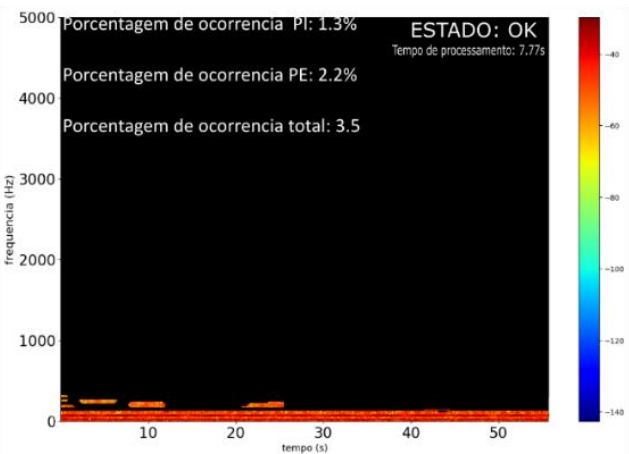

D- Audio 4

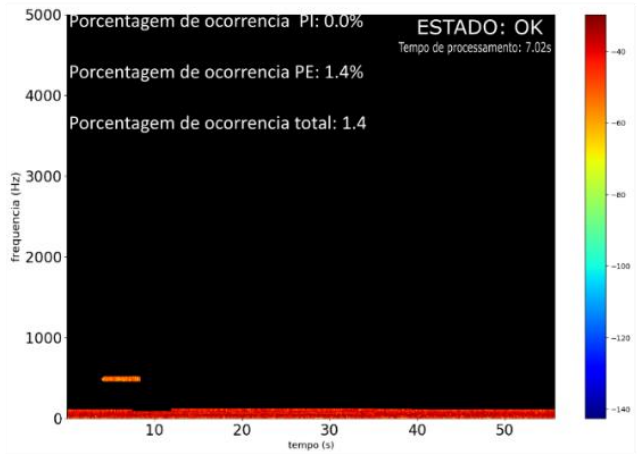

F- Audio 6

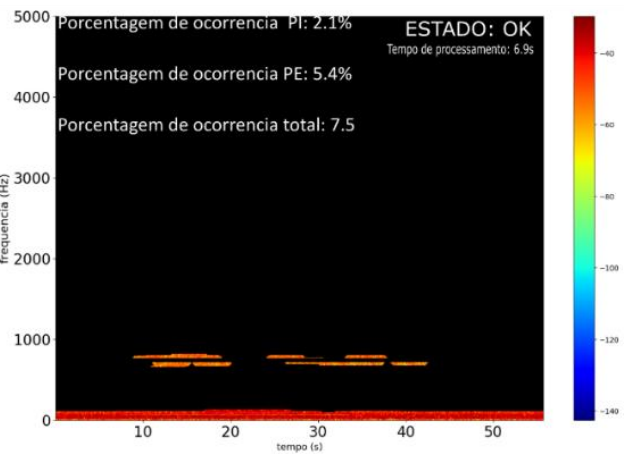

Fonte: Própria (2020).

Todos os diagnósticos apresentados na Figura 8 são referentes a rolamentos novos sem defeito. O tempo para gerar estes resultados é inferior (mais de 50\%) quando comparado ao tempo para gerar os resultados de um rolamento defeituoso, uma vez que não se utiliza a RNC para este diagnóstico. No momento da aquisição do sinal, foram impostas três situações de funcionamento do motor variando a carga mecânica sobre o eixo. Ainda assim, nas diversas situações imposta ao motor, $100 \%$ dos diagnósticos realizados foram corretos.

De forma semelhante, a Figura 9 demonstra o funcionamento do software para o diagnóstico de rolamentos com defeito na pista interna. 
Figura 09: Diagnóstico de um rolamento com defeito na pista interna.
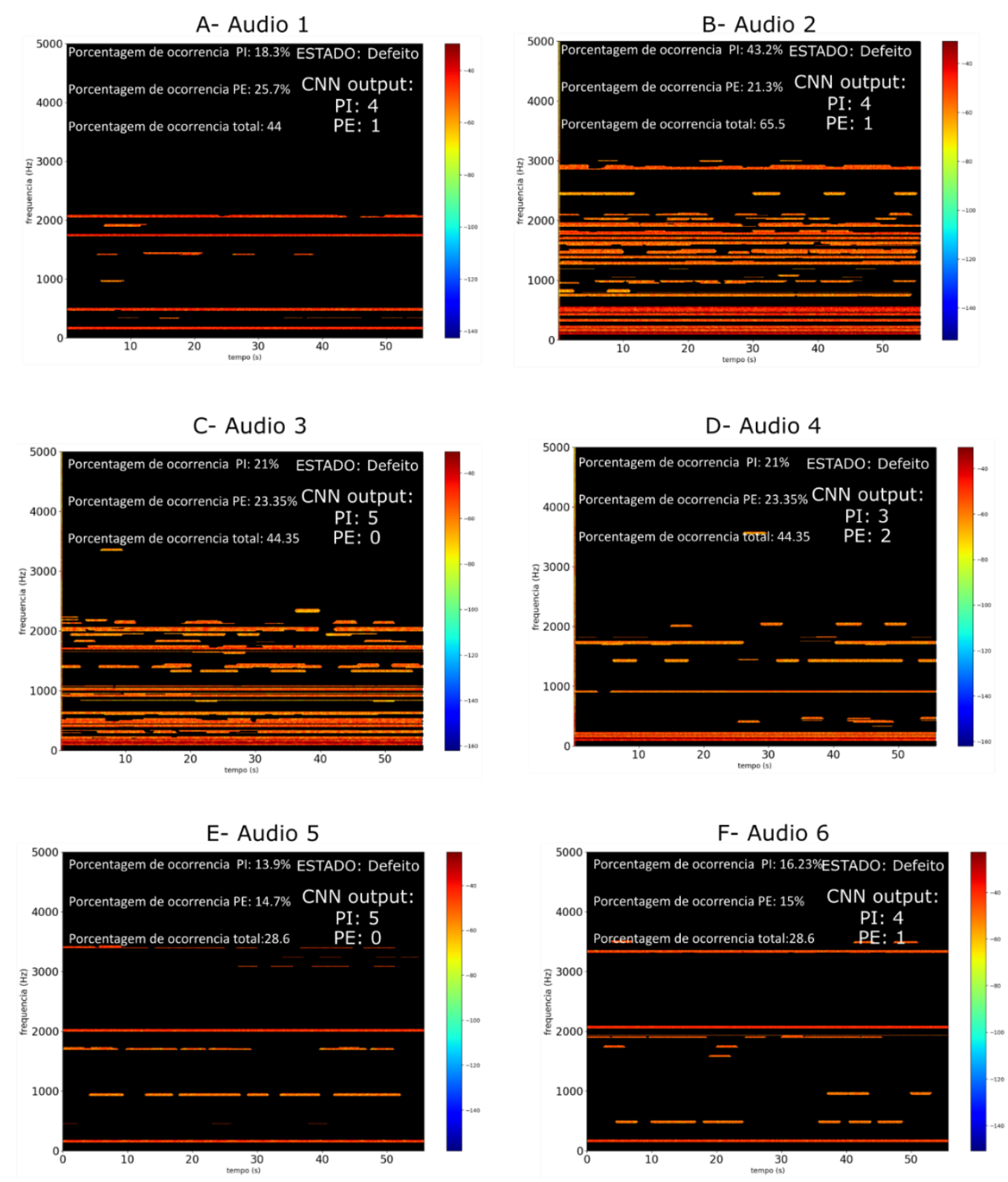

Fonte: Própria (2020).

O software implementado também obteve um desempenho satisfatório classificação de rolamentos com defeito na pista interna. Apesar de algumas imagens apresentarem um grau de certeza menor (menos segmentos classificados de forma certa) para todos os áudios correspondentes ao rolamento com defeito na pista interna, a classificação do mesmo foi realizada de forma correta.

Por fim, a Figura 10 apresenta os resultados que o software gerou ao inserir áudios de um rolamento com defeito na pista externa. 
Figura 10: Diagnóstico de um rolamento com defeito na pista externa.
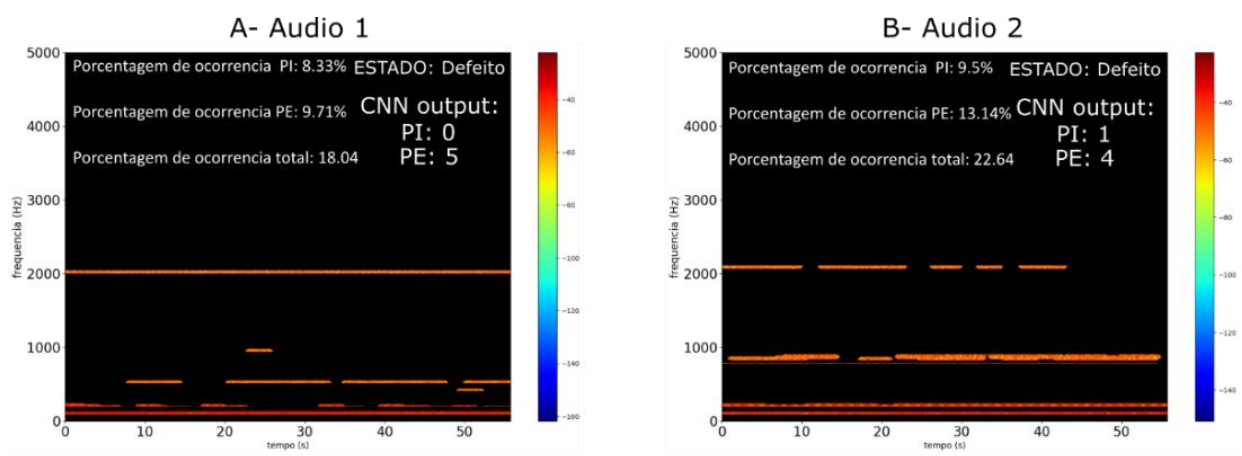

C- Audio 3
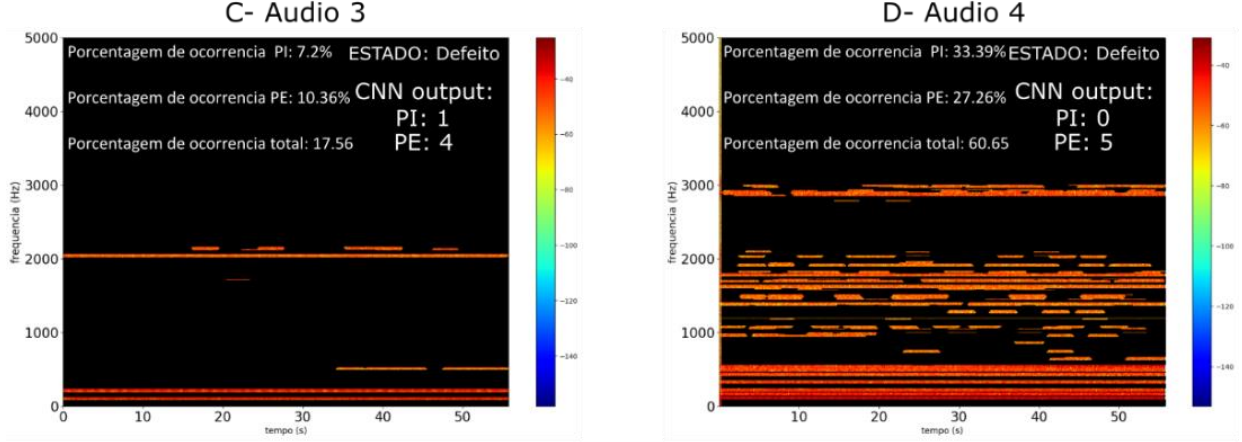

E- Audio 5

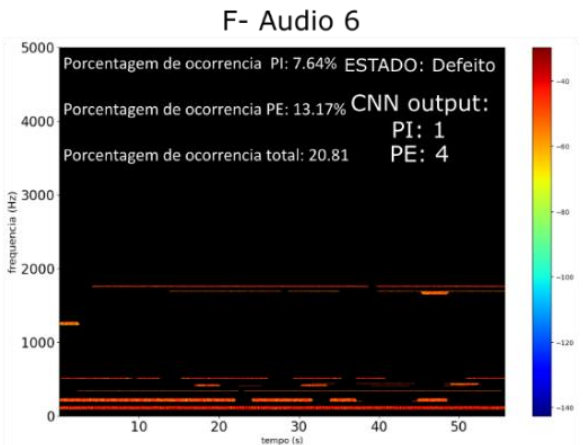

Fonte: Própria (2020).

De forma semelhante, o software implementado classificou todos os áudios desta classe de forma correta, com um tempo de processamento aproximado de 14s.

\section{CONCLUSÕES}

No estudo aqui apresentado foi desenvolvida uma ferramenta de suma importância para auxiliar o profissional responsável a diagnosticar de forma correta e rápida um rolamento em pleno funcionamento, independente da velocidade de funcionamento. Diferente das pesquisas realizadas tradicionalmente nesta área, o trabalho aqui desenvolvido utilizou um conjunto de técnicas para proporcionar um resultado objetivo, sem que haja necessidade de o operador deduzir informações. Com isto, além de facilitar a utilização da ferramenta, esta ferramenta confere maior confiabilidade no diagnóstico do rolamento. 
Inevitavelmente, o método de aquisição de sinal aqui utilizado está predisposto a ruídos externos, principalmente no âmbito industrial, onde há diferentes sistemas eletromecânicos em funcionamento continuo. Com isto, faz-se necessário para pesquisas futuras o desenvolvimento de um sistema de cancelamento de ruído, viabilizando assim a utilização desta ferramenta na esfera industrial.

Por fim, pode-se concluir que esta pesquisa possuiu uma relevância substancial, uma vez que foi capaz de diagnosticar os rolamentos de forma eficaz, mesmo utilizando componentes de baixo custo, quando comparado aos equipamentos convencionalmente utilizados para a mesma função.

\section{REFERÊNCIAS}

AHAMED, S. K.; KARMAKAR, S.; MITRA, M.; SENGUPTA, S. Novel Diagnosis Technique of Mass Unbalance in Rotor of Induction Motor by the Analysis of Motor Starting Current at No Load Through Wavelet Transform. 6th International Conference on Electrical and Computer Engineering, pp. 474 - 477, 2010.

AKBEN. "Acoustic spectral imaging and transfer learning for reliable bearing fault diagnosis under variable speed conditions", p. 1-19, 2019

GONZALEZ, Rafael C.; WOODS, Richard E. Processamento de imagens digitais. Editora Blucher, 2000.

HASAN, Md Junayed; ISLAM, MM Manjurul; KIM, Jong-Myon. Acoustic spectral imaging and transfer learning for reliable bearing fault diagnosis under variable speed conditions. Measurement, v. 138, 2019. p. 620-631.

Krizhevsky, A., Sutskever, I., and Hinton, G. E. Imagenet classification with deepconvolutional neural networks. Advances in neural information processing systems, 2012. p. 1097-1105.

NSK (org.). NSK Bearing Doctor: Diagnóstico Rápido de Ocorrências em Rolamentos, 2001. 36 p. Disponível em:

< http://www.nsk.com.br/upload/file/B08.pdf> Acesso em 05 nov. 2020.

PODDAR, S.; TANDON, N. Detection of particle contamination in journal bearing using acoustic emission and vibration monitoring techniques. Tribology International, v. 134, p. 154-164, 2019.

SALAZAR-VILLANUEVA, F.; IBARRA-MANZANO, O. G. Spectral analysis for identifying faults in induction motors by means of sound. In: CONIELECOMP 2013, 23rd International Conference on Electronics, Communications and Computing. IEEE, 2013. p. 149-153.

SANTOS, T. H.; GOEDDTEL, A.; SILVA, S. A. O.; SUETAKE, M. Scalar control of an 
induction motor using a neural sensorless technique. Electric Power Systems Research, 2014. Vol. 108.

SILVA, J. C. Sistema totalmente não-invasivo para determinação da velocidade de rotação do eixo, torque e rendimento em motores de indução em operação. 2018. $111 \mathrm{f}$. Dissertação (Mestrado) - Curso de Engenharia Mecânica, Centro de Tecnologia (ct) Programa de Pós-graduação em Engenharia Mecânica, Universidade Federal da Paraíba, João Pessoa, 2018. 